\title{
Socio-community insights into the COVID-19 pandemic in the cities of Abidjan and Bouaké (Ivory Coast)
}

(Aperçus sociocommunautaires de la pandémie COVID-19¹ dans les villes d'Abidjan et de Bouaké (Côte d'Ivoire))

\section{Dr. ADOU Paul Venance ${ }^{1}$, Dr. Fofana Valoua ${ }^{2}$, Dr. KRA Kouamé Chérubin ${ }^{3}$}

${ }^{1}$ Spécialité :Socio-anthropologie du développement, Enseignant-chercheur à l'Université Alassane Ouattara. ${ }^{2}$ Spécialité : Sociologie du développement, Enseignant-chercheur à l'Université Alassane Ouattara. ${ }^{3}$ Spécialité : Sociologie économique, Enseignant-chercheur à 1’Université Jean Lorougnon Guédé.

\begin{abstract}
A pandemic that appeared in Wuhan in China considered in the early stages of the spread as the disease of others goes, by its virulent spread, to modify the mores of different human communities. Now, all of humanity is struggling for its survival as the most vulnerable continent surprise's international opinion for its resilience while defeating the predictions of disasters. It is in this dispute that in Côte d'Ivoire, the taking in of this pandemic will experience two states. A clinical posture of curative and preventive regulation strongly represented in Abidjan while the localities of the interior escape the strictness of the provisions. As surprising as it may seem, COVID-19, which has become more visible among the Ivorian elite, will be weakly contracted and taken seriously by all communities. Although it is variously perceived by cultural areas, it still emerges a socially anchored resilience which nevertheless seems to be right in the spread of the pandemic. In a socio-anthropological approach, we will strive to elucidate the sociocommunity imaginaries that are the basis of the perception and community habits of COVID-19 in Côte d'Ivoire.
\end{abstract}

Keywords: social insights, community insights, pandemic, COVID-19, city of Abidjan, city of Bouaké

\section{Résumé}

Une pandémie apparue à Wuhan en chine considérée dans les débuts de sa prolifération, comme la maladie des autres, va de par sa virulente propagation modifier les mœurs des différentes communautés humaines. Désormais, l'humanité tout entière lutte pour sa survie tant bien que le continent le plus vulnérable surprend l'opinion internationale par sa résilience tout en déjouant les pronostics de catastrophes. C'est dans ce conteste qu'en Côte d'Ivoire, la prise en change de cette pandémie va connaître deux états. Une posture clinique d'une régulation curative et préventive fortement représentée à Abidjan tandis que les localités de l'intérieur échappent à la rigueur des dispositions. Aussi surprenant que cela paraisse, la COVID-19 qui s'est signalée bien plus dans la sphère de l'élite ivoirienne sera faiblement contracté et pris au sérieux par l'ensemble des communautés. Bien qu'elle soit diversement perçue par les aires culturelles, il en ressort tout de même, une résilience socialement ancrée qui pourtant semble avoir raison de la propagation de la pandémie. Dans une approche socio-anthropologique, nous nous évertuerons d'élucider les imaginaires

\footnotetext{
${ }^{1}$ COVID-19 est bien l'acronyme de l'anglicisme Coronavirus disease 2019. L'Académie française recommande que le mot COVID-19 soit précédé du déterminant féminin «la ». À cet effet, Monsieur Nicolas Martin, l'animateur de la chronique scientifique de cette prestigieuse institution fait, cette allocution : «(...) LA Covid-19. On me répète qu'il faut en parler au féminin, puisqu'il s'agit de l'acronyme de Coronavirus Disease 2019, traduit donc "Maladie à coronavirus 2019". Maladie étant féminin, je m'y tiendrai donc désormais : la Covid-19».
} 
sociocommunautaires qui fondent la perception et les habitudes communautaires de la COVID-19 en Côte d'Ivoire.

Mots clés : aperçus sociaux, aperçus communautaires, pandémie, COVID-19, ville d'Abidjan, ville de Bouaké

\section{Introduction}

La littérature sur la COVID-19 est abondante et témoigne d'un engouement réel pour la civilisation humaine en situation guerre ${ }^{2}$ contre une menace indomptable. Quel que soit le sens de la contribution intellectuelle abordé par les contributions interdisciplinaires pour remporter la bataille contre cette menace sanitaire pour la survie de l'humanité, nous décelons deux catégories de productions écrites. D'abord, celles des experts, professionnels et techniciens de la santé (Van Doremalen et al, 2020 ; Gautret et al, 2020 ; Liang et al, 2020 ; Deng et al, 2020 ; Flahaut Antoine, 2020; Chor-Cheug et al, 2020 ; Lorenzo d'Antigua, 2020 ; Arentz Matt et al 2020). Ensuite, celles des autres domaines connaissances scientifiques engagées de manière consciente à des contributions quelconques pour venir à bout de la pandémie. C'est bien dans ce sillage-ci que s'insère notre étude portant sur les perceptions sociocommunautaires relatives à la pandémie de la COVID-19 dans les grandes cités urbaines de la Côte d'Ivoire. Dans les villes d'Abidjan, Yamoussoukro et Bouaké, le brusque avènement de la grippe à coronavirus a dans un premier temps cristallisée la curiosité des citadins à propos de la nature de ce fléau. De cette situation, va naître deux appréhensions de la maladie. La première porte sur l'appropriation des conventions médico-légales promues par les autorités sanitaires. Très tôt, les citadins ${ }^{3}$ les plus avertis vont appliquer les mesures barrières dans le but de se prémunir de cette maladie inconnue. Dans un second temps, il sera constitué la frange des résistants contre la COVID-19. Pour une bonne part des habitants des grandes villes du pays, la pandémie est assimilée au «djè kouadio ». Pour eux, il n'est pas question de s'alarmer dans la mesure où la pharmacopée traditionnelle dispose de remèdes efficaces pour éradiquer les effets les sévères de la maladie. Ce système de croyance essentiellement basé les pratiques de la médecine indigénat va renforcer l'idéologie communautaire de la banalisation du fléau. Nous assistons par conséquence à la quasi-restauration du comportement habituel des citoyens aussi bien dans ces grandes cités urbaines que dans les autres villes et villages de la Côte d'Ivoire. De telles dispositions dans le système de santé mondiale pourraient-être assimilées à de graves dérives comportementales susceptibles de créer une crise sanitaire nationale. Pourtant, la réalité est toute autre, la COVID-19 n'a pu sévir de manière alarmante dans les différentes communautés constitutives du pays. Face à cette situation, nous sommes en mesure de poser la question suivante :

en quoi le système de croyance local a-t-il pu permettre aux communautés ivoiriennes de construire un caractère résilient vis-à-vis de la pandémie ?

Comment l'héritage culturel influence-t-il une bonne part des citoyens à définir leurs propres mécanismes de défense face à la COVID-19 ? Pour répondre à ces questions nous présenterons au préalable, l'objectif, la thèse et la méthodologie de travail.

L'objectif de cet article est de comprendre comment l'habitus dans le champ sociocommunautaire ivoirien détermine le citoyen en situation de la menace sanitaire du coronavirus.

\footnotetext{
2 "Nous sommes en guerre" : allocution du président de la République française Emmanuel Macron, à propos de l'épidémie de Covid-19 en France, ce lundi 16 mars 2020.

${ }^{3}$ Il s'agit ici d'une infime partie de la population issue en majorité de l'élite intellectuelle et politique du pays. Même au sein de celle-ci, le système de croyance local finira par prendre le pas sur les mesures barrières.

${ }^{4}$ Le « djè kouadio » n'est rien d'autre que la malaria. Mais, c'est une maladie perçue dans la conscience populaire comme l'effet immédiat d'une asthénie sévère.
} 
La thèse soutenue dans le cadre cet article est le suivant : l'attitude inconsidérée des citadins d'Abidjan et de Bouaké face à la menace réelle de la COVID-19 est intrinsèquement liée à leurs patrimoines socioculturels.

\section{Méthodologie}

Pour ce travail, nous nous retenons les deux plus grandes villes de Côte d'Ivoire. Ce choix est lié aux critères de représentation sociale qui nous prédispose à définir un ensemble de caractéristiques socioculturelles commune à ces deux localités, Abidjan ${ }^{5}$ et Bouaké ${ }^{6}$. Dans cette étude, nous avons eu recours à l'entretien semi-directif et à l'observation directe. Ces techniques de collecte de données nous ont permis d'interroger 57 personnes à Abidjan et 52 à Bouaké soit un total de 109 interrogées. Ces enquêtés sont regroupés en catégories sociales suivant : les Akan (27 personnes), les Krou (14 personnes), les mandés (23 personnes), les Gour ( 7 personnes), les Voltaïques (9 personnes) et les expatriés ( 29 personnes). Les principaux grands groupes sociaux des villes d'Abidjan sont représentés dans le staff des enquêtés ${ }^{7}$ dans le but de traduire objectivement l'opinion de ces derniers dans la restitution des résultats. L'ensemble de ces données est analysé selon la théorie de l'habitus et du capital culturelle (Pierre Bourdieu, 1972 : 272).

D'abord, nous présenterons le contexte de l'avènement de la COVID-19 dans le contexte des appréhensions sociocommunautaires des habitants d'Abidjan et de Bouaké. Ensuite, les résistances de ces citadins liées à leurs perceptions et attitudes en situation de la menace sanitaire. Enfin, nous ferons l'analyse de ces comportements des habitants de ces deux grandes villes dans la situation endémique.

\section{L'avènement de la COVID-19 et appréhensions sociocommunautaires des habitants d'Abidjan et Bouaké}

\section{I.1. Construction d'une imagerie sociale face à la primeur de la menace de la COVID-19}

En Côte d'Ivoire le premier cas de la COVID-19 est identifié le 11 mars 2020 à Abidjan. Ce cas d'infection va systématiquement déclencher une alerte d'urgence sanitaire qui va non seulement entraîner la prise des dispositions de quarantaine et sensibilisation des citoyens. Cette situation inhabituelle pour les ivoiriens va d'abord cristalliser l'attention des habitants d'Abidjan. Il s'en suit une vague de rumeurs ${ }^{8}$ qui va mobiliser le système de croyance ${ }^{9}$ local autour d'une menace qui selon les informations reconstituées ${ }^{10}$ s'apparente au paludisme. Selon PIERRE BOURDIEU (1980 : 88), il s'agit dans ce contexte d'un « Système de dispositions durables et transposables, structures structurées prédisposées à fonctionner comme structures structurantes, c'est-à-dire en tant que principes générateurs et organisateurs des pratiques et des représentations ». Dans la ville d'Abidjan, cette crise sanitaire va concerner au premier plan les autorités politiques ${ }^{11}$ et les

\footnotetext{
${ }^{5}$ Abidjan est la première grande ville du pays avec une forte population de 4707404 habitants (chiffres mis à jour récemment par le district d'Abidjan).

${ }^{6}$ Bouaké est la deuxième avec un taux de peuplement estimé à 608138 habitants. Ces deux cités urbaines présentent pratiquement les mêmes types d'habitants. Nous retrouvons ainsi environ $70 \%$ de nationaux et $30 \%$ d'expatriés (source statistique, INS). Ce qui est remarquable ici, c'est la revendication d'un lien d'appartenance socioculturelle propre à chaque groupement social. Surtout, propre à régir le comportement des citoyens dans la menace sanitaire de la COVID-19.

${ }^{7}$ Nos enquêtés sont issus de divers milieux sociaux dans ces villes. Ainsi, pour des raisons de confidentialité, ils seront cités sous le sceau de sobriquets. Dans cette étude, seuls les propos les plus avisés serviront à implémenter notre argumentaire.

${ }^{8}$ À partir des informations collectées par les voies de la presse locale (la télévision et les journaux), les symptômes de la nouvelle maladie «COVID-19» sont assimilés au paludisme ou «djè kouadio » en langue locale. Cette maladie est la résultante de fatigues excessives et toute une thérapie liée à la médecine traditionnelle y est appliquée. Aussi faut-il ajouter qu'à travers les réseaux sociaux, Whatsapp, Youtube et Tweeter toute une pharmacopée dérivée de recette de grande mère vient renforcer les croyances médicinales locales.

${ }^{9}$ Le système de croyance locale ici, n'est rien d'autres que l'ensemble des valeurs socioculturelles liées à la médecine traditionnelle enrichit de rumeurs (idem) liées à la COVID-19.

${ }^{10}$ À partir des informations officielles des autorités sanitaires, les communautés autochtones et allochtones et allogènes relèvent les symptômes comme la fièvre, les céphalées, les difficultés respiratoires et la toux comme similaires à ceux du «Djè Kouadio ».

${ }^{11}$ Parmi tant d'autres, le ministre de la Défense de l'époque, Hamed Bakayoko est frappé par la maladie COVID-19, le lundi 06 avril 2020.
} 
personnes nanties ${ }^{12}$ du pays. Une conscience plus avertie de la maladie est développée par cette catégorie sociale. Pour elle, la manière de penser la maladie est arrimée au standard officiel de $1^{\prime} \mathrm{OMS}^{13}$ qui consiste à se prémunir de la maladie par des gestes ${ }^{14}$ de distanciation sociale. Les habitudes d'échange social entre les individus de ce range seront considérablement modifiées désormais les salutations fraternelles, par les accolades et les baisers sont devenues pour eux des gestes dangereux qui peuvent être des vecteurs de contamination. La destruction du lien social basé sur l'interaction physique entre les personnes de l'élite social est proscrite. Monsieur MAMOUDOU, l'un de nos enquêtés issu de ce milieu déclare : «Notre vie en qualité de personne publique est devenue quasi infernale, nous ne pouvons plus aller au contact de nos partisans, les bains de foule et la convivialité qui animaient nos rencontres sont désormais frappé d'interdit. Il nous faut nous adapter et comprendre que le monde a changé ». À cet effet, il est remarqué dans cette société des huppés, une ambiance de «fin des temps ${ }^{15}$. Les meetings politiques, les concerts d'artiste et les soirées festives. Bref, toutes les activités publiques ou privés portant sur un rassemblement de plus de 50 personnes sont interdites. La vie sociale a rapidement flétri mettant ainsi la conscience collective au sein de ce groupe à un questionnement existentiel. Monsieur BETRI renchérit: "N'est-ce pas la fin de notre humanité ? Nous courons certainement à notre extinction. Demain, n'est plus certain, l'essence de la vie qui réside dans la proximité physique et les contacts corporelles a dorénavant disparu ». Au second plan, la vie pour les autres ${ }^{16}$ citadins n'a pas changé, la COVID-19 est répertoriée comme une menace sanitaire familière, proche du «Djè kouadio ». Surtout, depuis que cette maladie est déclarée, aucun membre de ce groupe social n'est infecté par la COVID-19. Monsieur KONAN déclare à juste titre: «Dans la pharmacopée traditionnelle Akan, il n'existe pas de maladie sans remède. Ce n'est pas le paludisme des blancs qui pourra nous effrayer. De plus, cette maladie est l'affaire des riches et ceux qui fréquentent les blancs. Depuis qu'ils ont annoncé leur COVID-19, je ne connais aucun proche qui en est victime ». L'état d'urgence sanitaire annoncé par les autorités et les mesures de distanciation sociale sont ignoré par les citadins dans les quartiers populaires, en occurrence dans les quartiers populaires d'Abidjan et Bouaké. Pour les habitants de ces lieux, la COVID-19 est une maladie importée qui ne s'attaque qu'à ce qui ont délaissé leur origine pour la vie des occidentaux. PIERRE BOURDIEU (1972:178-179), pense que ces représentations locales : "Fonctionnent comme une matrice de perceptions, d'appréciation et d'actions qui tend à reproduire les régularités immanentes aux conditions objectives de la production de leur principe générateur ». En plus, ils sont perplexes sur la contamination de cette maladie, surtout, qu'ils n'estiment qu'aucun de leurs proches cités dans les cas présentés dans les médias. En ce sens, le système de valeurs lié aux représentations sociales de cette pandémie, l'estime de soi et l'offre thérapeutique culturelle. D'abord, les habitants des communes populaires d'Abidjan affirment sans ambages que la COVID-19 est l'affaire de la classe des plus nantie. En effet, ils pensent que leurs habitudes, trop calquées sur le modèle occidental, font de ceux-ci, des êtres vulnérables en proie aux turpitudes du monde des «blancs ». Surtout, de par leur voyage à l'extérieur et relations d'affaires, ils ne peuvent que contracter la maladie. Ensuite, les citadins de ces circonscriptions s'identifient à l'homme d'origine ${ }^{17}$. Cette pensée de l'homme crée à l'image du Trèshaut, leur confère une confiance et une sérénité face à la menace. Ainsi, Monsieur KOUDOU PIERRE,

\footnotetext{
${ }^{12}$ Certains hommes du « showbiz » ivoirien et des acteurs de la scène diplomatique et politique national sont frappés aussi par la maladie à coronavirus. Leurs identités sont confidentiellement gardées par le secret médical.

${ }^{13}$ Organisation Mondiale de la Santé.

${ }^{14}$ Les gestes de distanciation sociale consistent au port du masque obligatoire, à se laver régulièrement les mains avec du savon ou la désinfecter avec du gel hydro alcoolique. Aussi, à tousser dans le creux du coude et observer une distance de sécurité d'au moins un mètre entre personnes.

${ }^{15}$ Comme le prédisaient les évangiles de l'Apocalypse de saint Jean.

${ }^{16}$ Les autres constituent l'ensemble des citadins qui ne côtoient pas le monde des personnalités politique et socioculturelles du pays.

${ }^{17}$ L'homme d'origine représente dans l'imagerie populaire. L'homme premier crée par l'Être suprême, l'humanoïde adamique sans aucune forme de mutation génétique. Dans le contexte de la COVID-19, il serait le plus résistant. De plus, ils l'affirment, «nous n'avons pas connu de cas coronavirus au sein de nos proches ». Dixit, AMADOU KEITA, l'un de nos enquêtés de Yopougon.
} 
déclare : «Nous sommes originaires d'Adam, le premier homme. Notre ADN et code génétique répondent de ce dernier. Pour preuve, les spéculations sur le sort de l'Africain voué à disparaître pour l'instant de cette crise sanitaire se rependent, mais la réalité est toute autre, nous nous portons mieux ». L'estime de soi de ces populations leur conduit à la promotion d'une identité originelle encrée dans les vestiges de l'histoire de l'humanité. Enfin, l'offre thérapeutique se décline par l'usage des plantes médicinales ${ }^{18}$ antipaludiques et antibiotiques. L'imagerie populaire conçoit la nature verte comme la source où regorge le remède à toutes les maladies de l'homme. Toutefois, s'il advenait que les plantes soient inefficaces aux pathologies humaines alors, la souche du mal serait donc non biologique. C'est bien cette pensée que Monsieur LAGO ${ }^{19}$ déclare dans son assertion : "Notre environnement, constitué de plantes, abrite le remède de tous les maux humains. S'il arrivait qu'une plante de source avisée soit inefficace face aux symptômes d'un mal qu'il est censé, guérir la source du mal serait ainsi crée par les expériences scientifiques de l'homme ». De manière voilée, dans la représentation culturelle, l'être humain reçoit tout ce qui assure son existence et sa vitalité de son biotope. Surtout, il existe dans cette perspective une interrelation entre les humains et leur milieu de vie. Alors «l'homme reçoit les soins, la nourriture gîte et couvert de la nature en retour, il retourne à la nature et celui-ci récupère tout ce qu'il lui avait donné ${ }^{20}$.

\section{I.2. Des manières de penser et d'agir des citoyens communautaires dans les villes d'Abidjan et Bouaké}

Le système de pensée sociocommunautaire repose sur l'héritage culturel, les emprunts liés au savoir-faire thérapeutique et aux informations du moment en rapport avec la COVID-19. Le schéma suivant illustre ces dispositions.

\section{Valeurs liées aux idéologies sociocommunautaires en situation de la COVID-19 à Abidjan et Bouaké}

Système de pensées

Socioculturelles

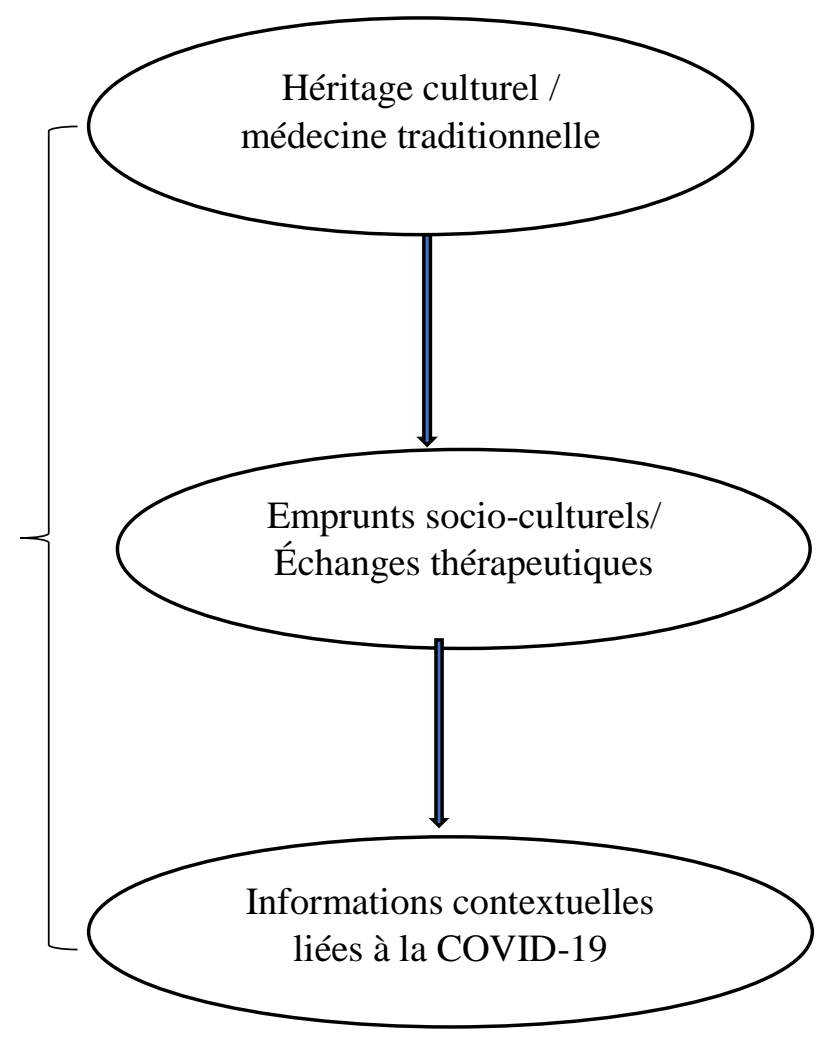

\footnotetext{
${ }^{18}$ Les feuilles de Nime, de l'Artemesia, de «DJEKA» (Alchornea cardifolia) sont parmi les plantes les plus prisées de de la pharmacopée traditionnelle. Surtout, utilisé à des fins préventives et curatives des symptômes ou pathologie de type bactériologique ou viral.

${ }^{19}$ Monsieur LAGO est l'un des représentants du conseil des tradipraticiens de Côte d'Ivoire.

${ }^{20}$ Monsieur LAGO, idem.
} 


\section{$\underline{\text { Explication du schéma }}$}

Le système de pensées socioculturelles repose trois paramètres de l'imagerie populaire : l'héritage culturel, les emprunts socioculturels et les informations contextuelles. D'abord, l'héritage culturel dans le contexte d'une maladie est tributaire des pratiques médicinales traditionnelles des principales aires culturelles Akan, Krou, mandé ${ }^{21}$ et Gours ${ }^{22}$. Dans ce registre, les pratiques sont constituées en connaissances enfouies dans les vestiges culturels de ces peuples et en savoir-faire liant la raison à la pratique. De surcroît, PIERRE BOURDIEU (1980 : 94) en déduit que «Les pratiques ne se laissent déduire ni des conditions présentes qui peuvent paraître les avoir suscitées ni des conditions passées qui ont produit l'habitus, principe durable de leur production ». Bref, chez les Akan, le médicament pour traiter des pathologies biologiques sont en premier lieu un don des esprits chtoniens. Ceux sont les esprits à l'origine de l'existence humaine qui délèguent aux humains une partie de leur trésor de vie par le biais des ancêtres leurs messagers. Monsieur KASSY, un guérisseur Akan, nous fait la confidence suivante : "L'ordre de la médecine chez nous, repose strictement sur les esprits créateurs. Ce sont eux qui transmettent aux initiés les plantes et les éléments utiles pour le traitement de toutes les maladies de notre société. Ils ont pour messagers les ancêtres ». À travers cette assertion, il faut comprendre qu'il n'existe aucun domaine de la santé de la communauté qui ne soit pas pris en charge par la médecine traditionnelle. Cette connaissance médicinale bien qu'ayant pour souche l'ésotérisme, est empiriquement matérialisée par l'usage des plantes pour les soins prodigués aux malades. Pour Monsieur BEUGRE, l'un des représentants des thérapeutes traditionnels de Bouaké, évoque le traitement d'un malade récemment pris en charge : «Dans le mois d'août 2020, j'ai reçu madame Akissi qui fut diagnostiquée malade de COVID-19. Seulement, avec la mixture et la décoction des plantes indiquées par les esprits. Ma patiente a recouvré entièrement la guérison ». Dans la tradition Krou, ce sont les esprits de la forêt qui transmettent aux guérisseurs l'antidote lié aux pathologies dans la communauté. Ici, il existe une reproduction sociale lié à l'activité de thérapeute traditionnel. En ce sens, les connaissances médicinales sont transmises d'une génération à une autre par l'initiation et l'enseignement. Toutefois, lorsqu'il advient des maladies de types endémiques où pandémique des rituels de purification sont organisés au sein de la caste des porteurs de masques pour évacuer le mauvais sort et permettre aux esprits de la forêt d'indiquer aux guérisseurs les remèdes appropriés. Monsieur ZAMBLE, un guérisseur Gouro de la commune de Yopougon, nous relate les dispositions traditionnelles en situation pandémique : «Depuis le mois de janvier 2019, le porteur de masque de notre village a reçu la révélation des esprits de la forêt de la menace d'un danger sanitaire à l'échelle mondiale. Nous avons ainsi procédé aux différents sacrifices de purification pour ainsi éloigner ce fléau de tous nos fils ». Il ressort de cette assertion la prédiction des alertes sanitaires et des actions mystiques et thérapeutiques menées à titre préventif pour prémunir les ressortissants de la communauté. Chez les Mandé et les Voltaïque, la tradition médicinale est liée au système d'apprentissage de certains initiés dans les sociétés secrètes dans les villages. Dans l'univers ésotérique de ces communautés, il existe plusieurs écoles initiatiques parmi lesquelles celles des secrets liés à la vie et à la mort. C'est dans cette école que les initiés reçoivent les indications sur le savoir-faire thérapeutique des maladies, mais aussi du traitement et de l'inhumation des morts. Parmi tous les initiés aux rites d'ascension sociale, quelqu'un est désigné par les esprits fondateurs de la société pour recevoir cette distinction de guérisseur. Monsieur KONE, un initié de ces rites, nous fait ces confidences : «Chez nous, les Dioula, la guérison des maladies est dévolue à une élite d'initiés choisie par les esprits fondateurs. Une fois investis, ces derniers peuvent prendre en charge tout type de maladies même, celles de type pandémique comme la COVID-19». Quel que

\footnotetext{
${ }^{21}$ Dans le contexte de cette étude, une extension est donnée à la connotation malinké comme étant une appellation regroupant non seulement les Mandés du Nord (Bambara et Dioula) mais aussi ceux du Sud (Dan, Wen, Toura, Gban et les Ben et Kweni).

${ }^{22}$ Les Gours regroupent dans le cadre de cette étude les Senoufos, Lobis, Koulangos. Précisons qu'ils ont une culture commune avec les malinkés. Selon les écrits historiques, tous sont issus de l'empire du Mali fondé par Soundiata Keita au XIIIe siècle.
} 
soit les aires culturelles, présentées, il existe un tandem entre le monde des esprits et des humains pour faire face aux maladies. Il existe fondamentalement des similarités systémiques dans le système sanitaire traditionnelle des communautés qui se côtoient dans les villes d'Abidjan et Bouaké. Cette réalité s'explique par les faits historiques liés à l'apogée de l'empire du Mali ${ }^{23}$ et au peuplement de l'actuel Côte d'Ivoire ${ }^{24}$. Dans ce contexte, des emprunts culturels d'ordre sanitaire vont caractériser les échanges de bons procédés entre ces différentes ethniques. C'est bien ce que confirme Monsieur Dyon, l'un des dignitaires des mandés du sud de Bouaké en ces termes : «Nous avons dans le cadre de nos alliances interethniques, empruntés des pratiques thérapeutiques avec nos frères Mandé du Nord et Gours. Aussi, nous les avons cédés bien de notre savoir-faire en la matière. Il nous faut reconnaître des similarités de part et d'autre ». Ainsi, il est indéniable qu'il existe une interaction des pratiques médicinales entre les communautés actuellement présente dans les villes d'Abidjan et Bouaké. Ce qui explique le partage entre eux des mêmes systèmes valeurs concernant la représentation de la maladie à coronavirus et leurs attitudes. Dans la conscience collective, les esprits créateurs par des rituels déjà réalisés aux origines ont activé le dom de protections des fils du terroir. De plus, les remèdes à tous les menaces sanitaires furent-ils, de type coronavirus sont à la portée des esprits créateurs. À cet effet, le comportement de non-respect des mesures de distanciation s'avère compréhensible. Monsieur TOPE exprime cette situation en ces mots : "Pour nous, africains, nous avons gardé notre lien originel avec nos ancêtres fondateurs et les esprits originels. Cet enracinement dans notre culture originel, nous confère une quiétude en ces temps de panique généralisée liée à la COVID-19. Nous conservons notre lien social avec nos origines et nos frères de même culture ».

\section{I.3. Registre des systèmes de valeurs culturels en confrontation avec les mesures conventionnelles de la COVID-19}

Dans la tradition des peuples vivant sur le territoire ivoirien, les mesures sociales sont essentiellement portées sur l'interaction entre les personnes dans le vécu quotidien. En ce sens, Monsieur TAPE, un enquêté ressortissant de l'ouest, affirme : «Chez nous, les Bété, comme chez l'ensemble des groupes ethniques du pays, la vie communautaire est fonctionnellement liée aux personnes qui composent la société. Surtout, les membres de la société doivent s'imbriquer mutuellement dans une proximité morale, physique et culturelle. Bref, l'homme dans notre société n'est jamais isolé du groupe ». Cette assertion laisse transparaître la substance de la vie communautaire chez les différents peuples de Côte d'Ivoire. Le contact social ${ }^{25}$ est le ciment de la vie dans les différentes aires culturelles du pays. Toutefois, depuis l'avènement de la crise sanitaire de la COVID-19, cette pratique sociale suffisamment encrée dans les mœurs des Ivoiriens se heurte au concept de distanciation sociale et des mesures barrières dans le contexte de la prévention de la maladie à Coronavirus. Le schéma suivant, juxtapose les valeurs traditionnelles de la vie sociale aux mesures conventionnelles de prévention de la pandémie. MICHEL CROZIER (1979:12), révèle à juste titre que : «Nous sommes prisonniers de notre milieu, des jeux que nous y jouons, de notre besoin d'exister pour, avec et contre autrui ».

\footnotetext{
${ }^{23}$ Se référer au commentaire sur le lien entre les Voltaïques et les Malinké, page 6.

${ }^{24}$ L'histoire du peuplement de la Côte d'Ivoire débute au XVIe siècle avec deux grandes vagues migratoires. Une partie des habitants de l'ancien empire du Mali (l'actuel Mali et Burkina-Faso) va descendre dans les territoires situés au sud, dans le nord de l'actuel Côte d'Ivoire. Une autre partie composée d'un groupe de ressortissants (actuel Akan) de l'empire du Ghana va progressivement occuper l'est, le centre et le sud. Les implantions de ces peuples ont été émaillées de conflits, mais des formes contrats sociaux furent contracter entre eux. Il s'agit ici d'échange de compétences notamment dans le domaine militaire, économique et sanitaire.

${ }^{25}$ Le contact social dans le cadre de cette étude, nous ramène au touché physique par la salutation, aux accolades et aux câlins qui sont des gestuelles teintées d'un symbolisme d'humanité, de respect et d'affection.
} 


\section{Comparaison de quelques pratiques sociales culturelles aux mesures de prévention de la COVID-}

19
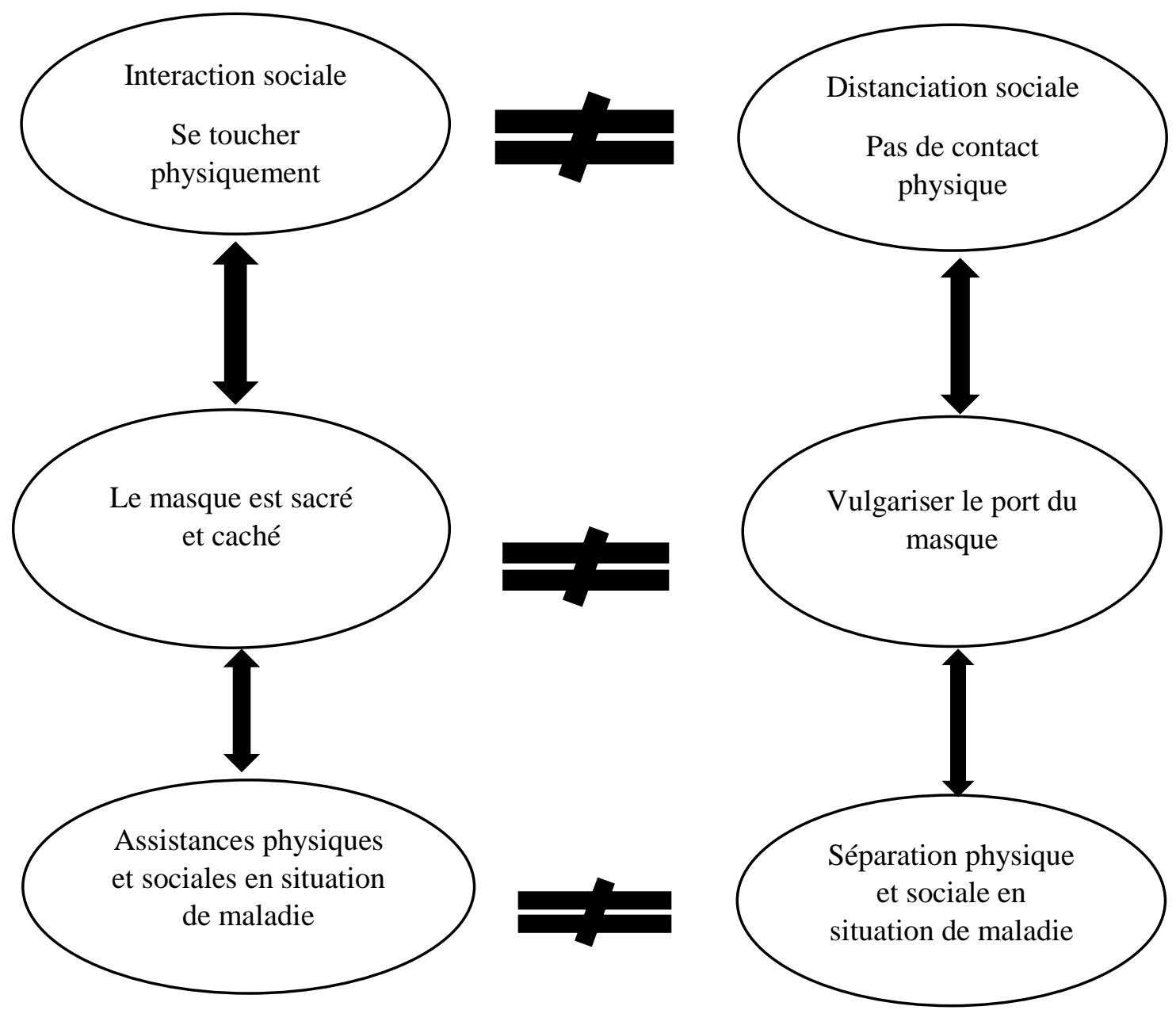

Sources, nos enquêtes, Novembre 2020

\section{Explication du schéma}

Dans cette représentation, la comparaison des éléments culturels et les actes édictés pour la prévention de la COVID-19 sont opposés. En premier lieu, dans la tradition des peuples étudiés, l'interaction sociale est le ciment de la vie communautaire. Pour ces groupes sociaux, le respect de la hiérarchie, la courtoisie et l'affection se traduise soit par l'échange de poignées de main, soit par des câlins et palpations. C'est bien cette réalité qu'exprime Monsieur AKA en ces mots : "Nos sociétés culturelles en Côte d'Ivoire sont régit par le principe d'échange physique dans la salutation, le respect des aînés et l'affection entre proches. Ces actes apparemment simples constituent le socle de l'organisation de nos sociétés traditionnelles ». Nous découvrons de cette affirmation l'importance du contact physique entre les individus d'une même société. Selon les propos de notre enquêté, la dynamique dans les rapports entre individus dans la communauté est fondamentalement basée sur les relations physiques. BOURDIEU (1987 : 24), analyse cette réalité comme 
un «Système de schèmes acquis fonctionnant à l'état pratique comme catégories de perception et d'appréciation ou comme principes de classement en même temps que comme principes organisateurs de l'action ». Par opposition, la distanciation sociale est l'acte primordial pour la prévention de la maladie. Une alerte sanitaire est en vigueur depuis l'avènement de la COVID-19, il s'agit de la proscription des contacts physiques entre personnes. L'auto isolement est encouragé entre les membres d'une même société. Cette ambivalence entre valeurs culturelles des aires ethniques en Côte d'Ivoire et les normes de prévention de la COVID-19 est l'une des causes du non-respect des mesures barrières liées à la prévention de la pandémie. Monsieur MOUSSA, un citadin de la commune d'Abobo renchérit: "Notre communauté ne saurait abandonner le touché, l'imbrication physique de ses membres pour le confinement comme prétexte de prévention d'une quelconque pandémie ». Selon cette assertion, le savoir-être dans la tradition des peuples de la Côte d'Ivoire s'oppose au plus haut point au concept de distanciation social propre à la protection contre la maladie à coronavirus. Par ailleurs, le port du masque est une coutume qui anime certains rituels chez les mandés du nord et sud du pays. Dans la tradition, l'imaginaire populaire conçoit le masque comme une entité sacré dont l'exposition est liée à des événements ésotériques. La présence du masque annonce l'initiation des néophytes à la scène de la maturité sociale. Elle exprime la conjuration d'un sort et la transmission d'un message des ancêtres et des esprits fondateurs. Le masque est donc assimilé au sacré. Il exprime crainte et respect des vivants. Pour ce faire, par l'observation des mœurs des Abidjanais et des habitant de Bouaké, il est inconcevable qu'une quelconque situation de crise puisse encourager la vulgarisation du port du masque. Ainsi, pour acter leur refus de souscrire à cette autre indication, les habitants de ces grandes villes de la Côte d'Ivoire ne porte pas de masques dans les lieux publics comme privés. Monsieur ZOUZOU, le responsable de la jeunesse du grand ouest de la commune Yopougon déclare en ce sens : "Le port du masque est une lourde responsabilité que nous ne saurions partager. Dans notre conscience, le masque est sacré, même si celui qu'on exige de porter ne correspond à aucun des nôtres, il n'est pas aisé non plus de briser en nous cette valeur enfouie ». La perception du masque est vue par les citadins deux ces grandes villes comme une institution sacrée. Tant bien même que l'ordre a été donné par les autorités sanitaires du port du masque obligatoire, cette mesure peine à être réalisé. Toutefois, il arrive que le port du masque soit partiellement respecté dans les services bureaucratiques et dans les supermarchés. Monsieur KONAN, un habitant de la ville de Bouaké, exprime son avis à ce sujet : «Nous sommes contraints dans certaines officines telles que les bureaux, banques et commerce à supporter le joug du port du masque. Aussitôt, dehors, nous nous en affranchissons ». Dans la tradition des communautés qui vivent dans les villes d'Abidjan et de Bouaké, la présence physique dans les situations de crise d'un parent est nécessaire. Surtout, lorsque le proche est soumis à l'épreuve de la maladie. Monsieur AKA, un ressortissant Akan de la ville d'Abidjan s'exprime dans ce sens : "Dans notre culture, nous considérons le malade comme un handicapé qui a besoin d'assistance pour assurer sa subsistance. La communauté lui apporte toute l'aide psychologique, physique et médicinale nécessaire à son rétablissement ». Dans la tradition, le patient ne saurait être isolé du groupe. Le rapprochement aux autres membres du groupe est donc nécessaire et vital. Par opposition, l'isolément, la mise en quarantaine sont recommandés en situation de contamination. Cette recommandation des autorités sanitaires se heurte aux pratiques des citadins d'Abidjan et Bouaké. En effet, ceux-ci sont toujours rattachés aux us et coutumes de leurs origines. Monsieur KOUDOU, un ressortissant Krou, s'exprime en ces termes : "Chez nous, les Bété, la notion de famille est suffisamment ancrée dans nos mœurs et ne saurait être assujettie à aucune réalité de notre vécu. Quand bien même qu'ils $s$ 'agissent de coronavirus, nous supporterons physiquement et moralement notre parent ».

II. Les perceptions et attitudes de résistances des habitants d'Abidjan et de Bouaké en situation de la menace sanitaire

\section{II.1. Les perceptions liées à la posture résiliente des citadins d'Abidjan et Bouaké}

Dans le cadre de notre étude, nous aborderons les perceptions sous l'angle socioculturel des aires ethniques. Il s'agit donc de la manière de penser et de commenter la COVID-19 par ces groupes sociaux. En ce sens, 
BOURDIEU $^{26}$ pense à juste titre le système de raison communautaire, comme un processus par lequel les valeurs acquises par la socialisation conditionnent le comportement des membres de chaque groupe. Ainsi, lorsque nous nous referons dans un premier cas, aux enquêtés du groupe ethnique Akan. Il ressort que la pandémie est répertoriée comme un châtiment des esprits à l'origine de l'existence humaine. En effet dans la cosmogonie Akan, il arrive les puissances chtoniennes réprime les dérives des hommes par des sanctions comme la maladie, la famille et les catastrophes naturelles. Selon l'imagerie populaire, les occidentaux ont certainement attisé par des interdits la colère des esprits. Monsieur KOUADIO, un notable du quartier Ahouanssou de Bouaké soutient: "Notre culture nous enseigne que les esprits créateurs veillent à l'équilibre des lois pour l'harmonie sur la terre des hommes. Ainsi, lorsque des principes sont scrupuleusement violés par les vivants, la colère de ces esprits s'abat sur eux». De plus, il situe les responsabilités et pense que «Les occidentaux avec le progrès de la science pense tout contrôler au point de tous se permettre. La pandémie est une punition les rappelant à l'ordre ». Dans un deuxième cas, les Mandé du Sud, viennent renchérir dans ce sens. Seulement, ils font la distinction des mondes. Pour eux, le monde des «blancs » ne peut être assimilé à ceux des «noirs » dans la mesure où la COVID-19 est une affaire des occidentaux. Dans la conscience collective quel que soit le type de civilisation les principes métaphysiques restent les mêmes. Alors, pour eux, les «blancs » ont énervé leurs propres dieux au point qu'ils doivent en payer le prix. Monsieur GUEI, un doyen d'âge, énonce les propos suivants : "Pour avoir réinventé tout ce que leurs dieux ont établi, ils ont cette fois-ci touché à quelque chose d'infâme. Par représailles, ils ont jeté l'opprobre sur eux et l'humanité tout entière ». Dans ce sens le malheur n'est pas que pour l'occident, mais si dans la perception des mandés du Sud, ça pourrait être eux les instigateurs des actes répréhensibles. Par conséquent, "Par la faute d'une civilisation, toute l'humanité est condamnée à payer un lourd tribut depuis l'avènement de cette maladie ${ }^{27}$. Dans un troisième cas, les mandés du nord saisissent la question de la pandémie liée à la COVID-19 comme une maladie ordinaire. L'influence de la culture mandingue et l'islam ont modelé le comportement autrefois animiste de ce peuple. En effet, le problème de l'existence humaine est désormais posé comme essentiellement d'ordre providentiel. En clair, les sinistres à l'origine des souffrances et les opportunités qui participent au bonheur dans la vie de l'homme dépendent du Très-haut. Alors, vivre dans cette perspective, c'est accepter avec sérénité toutes les situations qui meubles l'existence, en ce sens que l'être humaine ne peut y rien changer. Monsieur KASSOUM, un dignitaire de l'ethnie Mahouka de Bouaké, se prononce sans ambages à ce sujet : "Selon notre croyance, nous sommes sur terre semblable à des pèlerins dont les conditions de séjour dépendent du créateur. D’après, ces préceptes, l'homme ne crée pas les conditions de son existence. Il doit les apprécier comme grâce et bénir le créateur ». C'est bien cette croyance intériorisée qui conditionne l'indifférence que manifeste ce groupe social vis-à-vis de la menace réelle de la COVID-19. Toute cette manière de penser se traduit dans cette phrase : «COVID-19 ou pas, nous vivons les jours de notre existence avec la même reconnaissance au créateur. Pourquoi s'inquiéter des choses qui ne sont pas de notre volonté ». Dans le quatrième cas, c'est l'idée de famille qui est mise en avant dans la culture Krou. Ce groupe ethnique se perçoit comme une grande famille sur terre. Le lien d'appartenance à la communauté prévaut en toute situation. Dans les moments de bonheur et de malheur l'individu ne doit jamais se séparer de ses frères, ni montrer des signes de réserve qui pourraient violer son intégration. Dans cette même mouvance, les consignes de la distanciation sociale dans la menace sanitaire s'opposent à cet archétype culturel. Monsieur LAGO, l'un des responsables des doyens Bété de la commune de Yopougon, soutient cette thèse en ces propos : «Selon notre tradition, il est inconservable de souscrire aux types de mesures liées à la distanciation physique. Le rapprochement, le partage et l'assistance sont des valeurs de notre identité culturelle. En ce sens, comprenez que nous n'appliquons pas les mesures d'éloignement ».

\section{II.2. Des attitudes originelles des citadins des deux villes en situation pandémique}

\footnotetext{
${ }^{26}$ Bourdieu (1972 : 178-179), Op.cit

${ }^{27}$ Monsieur GUEI, idem.
} 
Les manières d'agir des habitants de la ville d'Abidjan et Bouaké sont tributaires de leurs points de vue culturels. En ce sens, nous avons répertorié quatre attitudes typiques aux différentes aires culturelles que nous avons enquêté. En premier, il s'agit, comportement des Akan face à la pandémie de la COVID-19. Des différents enquêtés que nous avons interviewé dans le cadre de cette étude, les Akan, paraissent les plus précautionneux face à la menace sanitaire. Ainsi, pour eux, une prise de conscience est nécessaire pour éviter de sombrer dans les comportements antisociaux tels que la rupture avec les membres de la communauté. Il s'agit de porter d'appliquer les mesures barrières et le port du masque que dans les espaces dédiés à la pratique par excellence de la culture occidentale. En effet, dans les services de l'État et les entreprises privées, le citadin doit se plier scrupuleusement aux règles de prévention édictées par l'OMS dans cette situation pandémique. Monsieur AKA, un ressortissant Akan, cadre de l'administration publique, nous décrit lui-même son comportement en ces mots : «Pas de mesures barrières entre mes parents, amis et moi, nous vivons nos habitudes originelles. Seulement, dans nos lieux de service et aux sites indiqués, nous appliquons strictement les recommandations des autorités sanitaires ». Il ressort des propos de notre enquêté, un comportement adaptatif propre à chaque lieu. Il s'agit pour lui, de conformer son comportement au gré des exigences des espaces consacrés. Tantôt, il est dans une logique de conservatisme lié au respect des percepts culturels. Tantôt, il agit en conformité avec les règles de mesure barrière. En deuxième, chez les mandés du sud, il est question d'agir selon la profondeur du lien social du ressortissant. Plus un individu est fortement ancré dans la tradition, plus, il sera affranchi du joug occidental donc, moins influencé par les mesures barrières édictées dans le cadre de la COVID-19. CAILLE (1992 : 203) explique en ce sens que «L'habitus est ainsi le résultat de cette alchimie qui nous conduit à tenir notre sort pour désirable, à croire que nous avons désiré et désirons être ce que, de toute façon, nous ne pouvons pas faire autrement que d'être ». Alors, dans ce cas de figure, le comportement du citadin est fonction de son rapport avec sa culture. À juste titre, Monsieur GEU Nestor, nous informe: "Je suis un dignitaire élevé en fonction des us et coutumes de chez moi. En ce sens, je ne me sens nullement pas concerné par les mesures barrières. Même s'il m'arrivait par contrainte d'y souscrire, cela reste isolé et occasionnel ». Par ailleurs, Monsieur GEU sylvain, rétorque: "Je n'ai pas la même assise culturelle que mon oncle. Je suis né à Abidjan alors j'applique les mesures de prévention dans la mesure de mes moyens sans toutefois déroger à l'idée de vivre en intégration avec mes proches ». Nos deux enquêtés ${ }^{28}$ ont des réactions propres à leur capital social. Le premier se situe dans une bulle culturelle qui est perçue par celui-ci comme un dôme qui le couvre. Tandis que le second accepte les prescriptions liées à la prévention. Toutefois, la résurgence de l'intégration communautaire l'emporte sur les efforts consentis pour être dans le conformisme propre aux mesures de prévention. En troisième, le comportement des mandés du nord se présente comme manifestement antagonique aux mesures barrières contre la COVID. Que ce soit dans la ville d'Abidjan ou Bouaké, les comportements des membres de ce groupe communautaire reste indépendant face aux exigences des autorités sanitaires. Rappelons, que pour ceux-ci les conditions de la vie ou du trépas sont fixées par le Toutpuissant. L'homme est soumis à la volonté, sur laquelle il ne détient aucune emprise. En ce sens, il devrait vivre librement sans se soucier du lendemain. Dans les villes d'Abidjan, ils vivent sereinement la période pandémique. Pour eux, pas question de s'avilir avec le port du masque et la distanciation physique. Monsieur ABDOUL nous exhorte en ce sens : "Pourquoi nous prendre la tête pour une pandémie qui ne dépend pas de nous. Selon notre culture et principes religieux, nous vivons chaque jour comme une bénédiction ». En quatrième, le comportement des Krou vivant à Abidjan et à Bouaké en situation pandémique est tributaire de leur croyance profonde aux liens de fraternité et de famille. Ainsi, les comportements recensés au sein des citadins originaires de ce groupe culturel traduisent toujours la primauté $\mathrm{du}$ rapprochement social au sein du groupe même en situation de COVID-19. Toutefois, la franchise culturelle est strictement dévolue aux membres la communauté et aux amis. Monsieur KOUDOU explique : «Je ne porte pas de masque et je ne m'éloigne pas de mes frères et amis pour cette histoire de COVID. Seulement, si je suis avec des inconnus alors je me soumets aux exigences des autorités sanitaires ». Nous

${ }^{28}$ Monsieur GEU Nestor et GEU Sylvain sont tous deux issus de la même famille. 
remarquons que par ces propos, l'enquêté trouve en la famille et dans son cercle d'amis une raison de quiétude spécifique à la tradition Krou. Alors, les mesures de distanciation physique et sociale sont tout simplement proscrites dans l'espace communautaire et amical.

\section{II.3. Des exceptions dans les perceptions et attitudes des citadins en situation pandémique}

La pandémie de la COVID-19 est certes perçue par les autorités ivoiriennes comme une alerte nationale pourtant, il existe deux pôles antagoniques dans l'appréciation de cet état de fait. Le premier, concerne la majorité des personnes qui prenne trop à cœur la menace au point qu'elle a modifié leur jugement de valeur et leur comportement. Cette frange est constituée en majorité par les personnalités politiques et les intellectuels ${ }^{29}$. D'abord concernant les autorités politiques, ils sont ceux-là qui constituent la ligne de front au premier plan dans la coopération internationale. Ils sont contraints par leur cahier de charge d'aller au contact des opportunités de développement vers les pays occidentaux. En ce sens, leurs fonctions sociales les obligent à faire fi de leurs convictions intérieures pour le respect scrupuleux de la nouvelle ligne de conduite liée aux mesures de prévention de la COVID-19. À ce titre, (BERNOUX, 1995 : 69), explique que «L'exercice de la fonction détermine le comportement beaucoup plus que les caractéristiques de l'individu». Ainsi, leurs perceptions de la pandémie est une réplique des considérations des averties personnes en occident. En clair, pour ceux-ci, la réalité pandémique est perçue comme une menace pour la survie de l'espèce humaine. Monsieur CISSÉ, nous fait cette confidence : "Notre niveau de responsabilité, nous oblige à faire fi de nos traditions pour aborder la menace sanitaire dans la dimension objective. Nous pensons tout comme nos frères occidentaux que la COVID-19 peut annihiler l'espèce humaine si nous ne changeons pas notre manière de penser ». De plus, nous observons une exemplarité dans l'application des mesures de prévention par cette élite. La distanciation sociale est observée de sorte que les contacts physiques par l'échange de poignées de main ou les accolades sont proscrites. Surtout, le port du masque est constamment respecté. Particulièrement, dans la posture au service, l'ardeur est redoublée. Monsieur DIOMANDE, nous expose les raisons de ce comportement : "Il faut dire que même parmi nous au départ, certain ont douté de la pandémie. Mais, la contamination et le décès de certains de nos collègues et collaborateurs nous ont convaincu à l'adoption d'une posture plus sérieuse ». En fait, la conscience de la réalité COVID n'était pas essentiellement tributaire de la sagesse de ces élites, mais aussi des expériences qu'ils ont traversées. Par ailleurs, le deuxième cas de figure à trait aux citadins qui vivent dans les villages périurbains de dans les villes d'Abidjan et Bouaké. En ces lieux, nous observons que les habitudes n'ont pas été altérées par les dispositions préconisées dans le cadre de la prévention liée à l'avènement de la pandémie. La distanciation sociale et le port du masque sont restés lettres-mortes. Monsieur AGOU, s'explique à juste titre : «Je ne vois pas une raison de me préserver de la COVID-19. En effet, mon champ de relation et mes habitudes de travail sont strictement éloignés de la scène politique et intellectuelle des élites du pays ». Selon les propos de notre enquêté, la distanciation sociale et professionnelle avec le monde élitiste affranchit de la menace sanitaire, comme il le renchérit : «C'est la maladie des riches et de leurs amis ».

\section{Analyse des insuffisances systémiques et des comportements des citadins d'Abidjan et Bouaké dans la situation pandémique}

\section{III.1. Des limites des systèmes de valeurs locaux}

Les sociétés culturelles de la Côte d'Ivoire partagent les fondamentaux d'un même rituel social et religieux. En ce sens, la crise sanitaire liée à a la prolifération des zones de contamination dans les différentes zones du monde, vont d'une part mettre à rude épreuve le système de valeur ésotérique des populations urbaines d'Abidjan et de Bouaké. Dans la tradition relative aux pratiques sanitaires des aires culturelles que nous retrouvons dans les deux villes étudiées, le système de croyance ésotérique demeure le socle. Ainsi, dans l'imagerie populaire, la couverture sanitaire est l'apanage des esprits créateurs et des ancêtres. ZALEZNIK,

\footnotetext{
${ }^{29}$ Les intellectuels dans le contexte ici sont pour une bonne part ceux qui ont fait leurs études dans les pays occidentaux.
} 
(1994:4), enseigne dans ce contexte que "L'inconscient est un facteur déterminant (...) le passé d'un individu est un facteur déterminant de son comportement ». Les entités originelles, transmettent aux vivants un ensemble de prescriptions et de remèdes qu'ils se doivent de strictement respecter pour assurer leur bienêtre. La croyance est bien le moteur de la protection sanitaire des membres du groupe social. Toutefois, la COVID-19, ne touche pas que les personnes nanties ou en proximité avec ceux-ci. Elle est bien une pathologie comme toute autre maladie scientifiquement détectable. Pour Monsieur Koffi, un membre de conseil des tradipraticiens d'Abidjan : «La COVID-19 est une variable encore inconnue de notre système sanitaire. C'est ce qui peut expliquer les quelques cas de maladies parmi nous. Mais tout est mis en ouvre par les rituels pour y remédier ». Selon les propos de notre enquêté, le facteur de vulnérabilité causé par la maladie à coronavirus sera effectivement traité par une ordonnance prescrite par les mannes. Ainsi, l'itinéraire thérapeutique socioculturel dans les villes d'Abidjan et Bouaké obéit au principe du respect de l'ordre originel. Il s'agit ici de la cosmogonie relative aux origines de ces peuples et leurs rapports avec l'être suprême. De ce rapport entre hommes et esprits, réside le remède à tous les maux sociétaux. Dans cette logique, l'avènement de la COVID-19 s'explique par des disfonctionnements générés par le nonrespect des préceptes de vie tels que stipulés par les ancêtres et les esprits créateurs. Monsieur MAMADOU, un tradipraticien de la ville de Bouaké renchérit: "Si les hommes respectaient les lois de la nature telle que prescrit par nos ancêtres, le monde se porterait bien et nous ne souffrirons plus de maladies de type pandémique, comme la COVID-19». Par ailleurs, bien que nous retrouvons dans les villes étudiés des peuples fort attaché à leur culture, cela n'empêche pas la pandémie de se propager insidieusement en leur sein. Chaque jour, dans le rapport sanitaire officiel, des cas de contamination sont déclarés au journal télévisé de vingt heures. Parmi ces cas révélés, figure aussi bien les plus nantis que les plus pauvres. La maladie ne prend donc pas en compte les considérations sociocommunautaires. Monsieur ELVIS, un responsable du service d'infectiologie au CHU de Treichville à Abidjan, nous informe à ce titre : « $L a$ maladie à coronavirus avait débuté avec des cas de personnes plus ou moins nantis, mais depuis la fin de l'année dernière jusqu'à présent les données ont changé. Nous enregistrons maintenant des cas de plus en plus nombreux au sein de la population à revenu moyen et défavorisé ». Cette révélation indique que le système de croyance et des valeurs socioculturels à certes le mérite d'affranchir les communautés vivant dans les grandes villes de Côte d'Ivoire d'une psychose généralisée, mais il l'expose aussi à la contamination directe. En effet, la tradition de ces citadins est aux antipodes des mesures de prévention telle que nous avons pu le démontrer antérieurement.

\section{III.2. Des limites du système de prévention des autorités sanitaires}

Le système prévention des autorités sanitaires s'articule autour Conseil National de Sécurité (C.N.S.). Cette institution est présidée par le chef de l'État et est composée des experts et des praticiens du domaine de la santé. Elle a d'abord eu le mérite de lancer l'alerte nationale sur l'avènement de la pandémie de la COVID19 dès le lundi 16 mars 2020. L'essentiel des mesures prises pour prémunir la population s'est décliné en treize points. Parmi ce chapelet de mesures de prévention, trois mesures seulement ont pu résister à la manière de penser et d'agir propre aux communautés vivant dans les villes. Il s'agit entre autres de la gratuité totale du diagnostic, de la prise en charge de tous les cas suspects et confirmés de COVID-19, de la mise en quarantaine des cas suspects et des personnes ayant eu un contact avec les malades. Concernant les autres directives, elles peuvent être classées comme étant des dispositions prises soit pour équiper et gérer à une période bien déterminer la menace sanitaire. Même pour des mesures impliquant les responsabilités des citoyens, l'engouement des populations dans ces grandes villes reste assujetti à leurs systèmes de valeur culturel. En dépit des efforts d'informations et de sensibilisation effectuée par l'État et le collège des agents de santé, les habitudes semble toujours prendre le pas sur les comportements et gestes recommandés. BOURDIEU (1980:94), déclare à cet effet que, "Les pratiques ne se laissent déduire ni des conditions présentes qui peuvent paraître les avoir suscitées ni des conditions passées qui ont produit l'habitus, principe durable de leur production ». La télévision est l'une des tribunes officielles la plus usitée pour la sensibilisation. Tandis que, les citadins d'Abidjan et de Bouaké n'ont que de ce canal leur propre conception. Monsieur ADAMA, l'un des responsables de communauté en fait l'expression: "À travers la télévision, nous attendons toujours des discours trop 
intellectuels des bonnes mours à avoir pour ne pas être victime de la COVID-19. Mais la majorité de nos concitoyens sont peu alphabétisés pour attendre le message. Surtout, pour eux, il s'agit toujours d'une maladie des occidentaux ». Même si, au point treize des mesures édictées, l'ordre est donné par le chef de l'État aux chefs traditionnels de relayer le message de sensibilisation. Néanmoins, il demeure dans les faits, que ceux-ci ne partagent pas le but de la mission. Monsieur AKA, l'un des chefs de la communauté Akan d'Abidjan, s'exprime en ces mots: «Nous sommes chefs et garant de valeurs culturelles alors, comment pouvons-nous faire fi de ce qui définit notre identité pour enseigner autres choses. Par formalisme, nous relayons le message de nos autorités, mais nous n'en avons pas réellement la conviction ». En clair, il apparait, une forte résistance des communautés au changement de comportement lié la prévention du COVID-19. Pour eux, l'appropriation et la mise en œuvre des mesures barrières de sécurité sanitaires nécessaires pour éviter la maladie porte atteinte à leur intégrité socioculturelle et à leurs identités. Ainsi, il ressort que le système actuel de prévention ne tient pas compte des spécificités culturelles propres à chaque communauté et catégorie sociale. Alors, les citadins semblent ne pas se reconnaître dans la promotion des mesures de prévention et préfèrent demeurer dans leurs traditions.

\section{III.3. Des limites liées au niveau de vie des citadins d'Abidjan et Bouaké}

Dans les quartiers populaires de la ville d'Abidjan et de Bouaké, les différents groupes identitaires vivent dans des conditions de vie souvent précaire. En effet, cette population tire sa subsistance d'une économie informelle. Surtout dans les quartiers populaires d'Abobo, Yopougon et Adjamé, les activités commerciales fleurissent à tout point de rue pour répondre aux besoins primaires des citadins. Bref, le niveau de vie des habitants des deux grandes villes est relativement faible et essentiellement axé sur la satisfaction des besoins alimentaires, le logement et le déplacement. Les ressources financières se font rares au point que les besoins de soins et d'équipement sont relégués à la dernière des préoccupations quotidiennes. CROZIER (1979: 12), affirme sans ambages que «Nous sommes prisonniers de notre milieu, des jeux que nous y jouons, de notre besoin d'exister pour, avec et contre autrui ». Alors, dans cette situation de crise économique traversée par les communautés des villes d'Abidjan et de Bouaké, l'avènement de la pandémie exige un comportement hygiénique et de prémunition qui ne demeure pas sans coût financier. En ce sens, l'achat des savons, des gels hydroalcooliques, des masques de protection vient alourdir le carnet des dépenses journalières des citoyens déjà excessivement éprouvés. Monsieur KODJO, l'un des chefs de quartiers de Bouaké, nous décrit cette situation en ces mots : «Voici que nous sommes dans une situation de disette. Nous peinons à assurer notre pitance quotidienne et les autorités nous demandent de prendre en charge l'achat des équipements pour la prévention du nouveau coronavirus. À dire vrai, en dehors de nos valeurs culturelles, nous en sommes incapables ». L'enquêté, révèle que la croyance en la tradition pour les moins nantis est le palliatif à cette situation de crise. Bref, les prescriptions sanitaires dans la coutume selon les diversités culturelles n'exigent aucun investissement financier. Dans l'imagerie populaire, "Les divinités ne permettraient pas qu'un fléau venu de d'autres cieux, décime leurs rejetons, c'est cette réalité qui nous maintient gracieusement en vie malgré les prédictions funestes de l'OMS au sujet la situation de la COVID19 en Afrique $»^{30}$. Aussi, dans les services, les dispositions prises pour la sécurisation sanitaire des usagers se heurtent à des habitudes socialement ancrées. En effet, le masque devient dans certaines situations, un simple apparat dédié à couvrir le menton. Les mains lavées à l'entrée des officines sont aussitôt au contact du nez est des visages souvent découverts de masques. Madame KADY une conseiller clientèle de supermarché de la ville de Bouaké nous relate le comportement des usagers en ces termes : «Tantôt, vous apercevez certains clients avec leurs caches nez sur le menton, les mains déjà lavées à l'entrée qui traîne sur le visage et souvent au contact de la bouche et du nez. À chaque fois que nous les abordons, ils disent être étouffés par le masque».

\section{Conclusion}

${ }^{30}$ Idem, Monsieur KODJO 
En définitive, il apparaît dans cette étude une configuration communautaire de la pandémie de la COVID-19 dans les deux plus grandes villes de la Côte d'Ivoire. De toutes les aires culturelles qui peuplent la ville d'Abidjan et de Bouaké, une seule manière de pensée la maladie est représentée. La maladie à coronavirus est un fléau importé. Une conception bien enracinée de la pandémie qui puise ses sources dans la cosmogonie des traditions du système de santé sociocommunautaire. Les citadins dans ce contexte expriment leur posture originelle sans tenir compte des nouvelles mesures préventives des autorités sanitaires du pays. Cette situation dispose à la prépondérance du mode de vie communautaire à celui du pouvoir publique dans la situation de la menace sanitaire de la COVID-19. La théorie de l'habitus et du capital culturel nous ont permis d'abord de comprendre que l'attitude des populations dans les villes d'Abidjan et Bouaké en situation pandémique est tributaire de la socialisation typiquement liée aux préceptes socio-sanitaires. Ensuite, par le capital culturel, les citadins d'Abidjan et Bouaké présentent les dispositions du système de santé communautaire comme gage de préservation de la COVID-19. Alors pourquoi semble-t-on pensée que la modernité a-t-elle pu altérer définitivement la personnalité culturelle des citadins dans les nouvelles sociétés africaines?

\section{Bibliographie}

1. AURIOL J. (1994), Approches cognitives de la décision et représentation sociale, Revue Internationale de Systémique, vol. 8, N² 2, pp. 139-166.

2. AURIOL J. (1995), Décision et action stratégique : les apports de l'approche socio-cognitive, Revue de Gestion des Ressources Humaines, $N^{\circ} 17$, novembre, P20.

3. BAUER M. (1993), Les patrons de PME entre le pouvoir, l'Entreprise et la Famille, Interéditions, P512.

4. BERNOUX P. (1995), La sociologie des entreprises, Éditions du Seuil, P373.

5. BOUDON R. (1995), Le juste et le vrai : Études sur l'objectivité des valeurs et de la connaissance, Fayard.

6. BOURDIEU P. (1972), Esquisse d'une théorie de la pratique, Librairie Groz, P223.

7. BOURDIEU P. (1979), La distinction : critique sociale du jugement, Les Éditions de Minuit, P672.

8. BOURDIEU P. (1980), Le sens pratique, Les Éditions de Minuit, P480.

9. BOURDIEU P. (1980/a), Questions de sociologie, Les Éditions de Minuit, (édition aug. d'un index 1984), P271.

10. BOURDIEU P. (1987), Choses dites, Éditions de Minuit, P228.

11. CROZIER M. \& FRIEDBERG E. (1977), L'acteur et le système, Éditions du Seuil, P445.

12. MUCCHIELLI A. (1992), L'identité, P.U.F., Collection « Que-sais-je ? », P128.

13. ZALEZNIK A. (1994), Les ressorts de l'action : Freud et la conduite des entreprises, Interéditions, P296. 